\title{
Caracterização do trabalho de enfermeiros e profissionais do Núcleo de Apoio à Saúde da Família na Atenção Primária
}

\author{
Characterization of work of nurses and health professionals \\ in Support Center for Family Health in Primary Health Care
}

Dixis Figueroa Pedraza ${ }^{1}$, Daiane de Queiroz², Márcia Cristina Sales ${ }^{3}$, Tarciana Nobre de Menezes ${ }^{1}$

${ }^{1}$ Programa de Pós-Graduação em Saúde Pública, Universidade Estadual da Paraíba (UEPB) - Campina Grande (PB), Brasil.

${ }^{2}$ Faculdade de Ciências Médicas de Campina Grande (FCM) - Campina Grande (PB), Brasil.

${ }^{3}$ Universidade Federal de Rio Grande do Norte (UFRN) - Natal (RN), Brasil.

DOI: http://dx.doi.org/10.7322/abcshs.v43i2.993

\begin{abstract}
RESUMO
Introdução: Avaliar o trabalho dos profissionais de saúde da atenção básica pode revelar informações importantes relacionadas à forma de produção de cuidado, essenciais para sua qualificação e atendimento das necessidades de saúde da população. Objetivo: Este estudo se propôs caracterizar o trabalho de enfermeiros da Estratégia Saúde da Família e profissionais de saúde de Núcleos de Apoio à Saúde da Família em um município da Paraíba, Brasil. Métodos: Trata-se de um estudo transversal no qual foram incluídos enfermeiros da Estratégia Saúde da Família e profissionais de saúde dos Núcleos de Apoio à Saúde da Família da cidade de Campina Grande, totalizando 52 profissionais, 20 enfermeiros e 32 de outras formações da área de saúde. Utilizou-se um questionário estruturado com informações sobre formação profissional, características do trabalho e satisfação profissional. O projeto teve aprovação pelo Comitê deÉtica em Pesquisa da Universidade Estadual da Paraíba. Resultados: Os enfermeiros destacaram falta de solidariedade no trabalho e de reconhecimento. A maioria dos profissionais dos Núcleos de Apoio à Saúde da Família mostrou insegurança decorrente de instabilidade laboral e medo de ficar sem trabalho. Ambos os grupos de profissionais indicaram baixa satisfação com a estrutura física, o atendimento individual à demanda e as reuniões com a comunidade. Conclusão: Os profissionais da Atenção Primária à Saúde do município têm que lidar com limitações impostas por um cenário estrutural pouco favorável das unidades de saúde e vínculos trabalhistas marcados por insegurança, falta de solidariedade e baixo reconhecimento.
\end{abstract}

Palavras-chave: Atenção Primária à Saúde; saúde da família; condições de trabalho.

\begin{abstract}
Introduction: Assessing the work of primary care health professionals can reveal important information related to the way of providing care, essential for their qualification and meeting the health needs of the population. Objective: This study aimed to characterize the work of nurses in the Family Health Strategy and health professionals in the Centers for Family Health Support in a city from Paraíba, Brazil. Methods: This was a cross-sectional study in which nurses from the Family Health Strategy and health professionals from the Family Health Support Centers of the city of Campina Grande were enrolled, comprising 52 professionals, 20 nurses and 32 from other health training. A structured questionnaire with information about professional formation, working characteristics and professional satisfaction was used. The project was approved by the Research Ethics Committee of the State University of Paraíba. Results: Nurses highlighted lack of solidarity and recognition at work. Most professionals from the Centers for Family Health Support showed uncertainty due to work instability and fear of being dismissed. Both professional groups expressed low satisfaction with the physical structure, the unaccompanied attention to population and meetings with the community. Conclusion: The city professionals of Primary Health Care must deal with restrictions imposed by structural problems in the health units and labor bonds marked by insecurity, lack of solidarity and low recognition.
\end{abstract}

Keywords: Primary Health Care; family health; working conditions. 


\section{INTRODUÇÃO}

A proposta da Estratégia Saúde da Família (ESF) representa o modelo assistencial brasileiro para Atenção Primária à Saúde (APS) como forma de reorganização dos serviços e de reorientação das práticas profissionais ${ }^{1}$. Está implantada em todo país e representa a porta de entrada prioritária do Sistema Único de Saúde (SUS) ${ }^{1}$. Tal processo gera uma transformação do modelo sanitário tradicional biomédico, com ações curativistas e individuais, para uma assistência centrada na família e na comunidade de forma integral e contínua, focada em ações de promoção, prevenção e recuperação $0^{1-3}$.

A ESF, além do médico, auxiliar de enfermagem e agente comunitário de saúde, tem no enfermeiro um importante membro da equipe básica multidisciplinar, por ser este o profissional que é elo ativo no processo de consolidação da estratégia como política integrativa e humanizada da saúde. O enfermeiro tem grande importância na equipe multiprofissional de saúde no contexto da ESF, com atribuições como a supervisão e ampliação das atividades dos agentes comunitários de saúde e dos auxiliares de enfermagem ${ }^{3}$. Ainda, esse profissional deve estar provido de características como liderança, bom relacionamento, comprometimento e postura ética que podem ser indispensáveis nas suas práticas multidimensionais de cuidado e gerenciamento dos serviços de saúde conforme exigências atuais ${ }^{4,5}$.

Apesar dos avanços alcançados na APS associados à implementação da ESF, reconhece-se que seus princípios e práxis ainda não representam a realidade no cotidiano dos serviços de saúde, sobretudo em relação à integralidade do cuidado, com importantes desafios no que se refere ao perfil dos profissionais e ao trabalho em equipe ${ }^{6}$. Dessa forma, a criação dos Núcleos de Apoio à Saúde da Família (NASF) foi concebida como forma de apoio especializado do trabalho das equipes de saúde da família por profissionais de diferentes áreas do conhecimento, propiciando maior abrangência, resolutividade, territorialização, regionalização e ampliação das ações e competências relacionadas aos problemas clínicos e sanitários ${ }^{1,2}$. Considerando o trabalho em saúde enquanto constituição de saberes e práticas, sua avaliação torna-se importante na medida em que pode revelar a forma de produção de cuidado ${ }^{5}$. Nesse sentido, o conhecimento da prática do dia a dia dos profissionais é fundamental para alcançar a qualificação do cuidado e o atendimento das necessidades de saúde da população ${ }^{7}$.

Cabe acrescentar que apesar dos profissionais de saúde da APS no Brasil constituírem um enorme contingente de força de trabalho no sistema de saúde, suas condições e satisfação relacionadas ao trabalho ainda merecem maior quantidade de estudos ${ }^{8}$. Com base nestes pressupostos, este estudo se propôs caracterizar o trabalho de enfermeiros da Estratégia Saúde da Família e profissionais de saúde de Núcleos de Apoio à Saúde da Família em um município da Paraíba.

\section{MÉTODOS}

Este estudo faz parte da pesquisa intitulada "Atenção Básica à Saúde no município de Campina Grande, Paraíba: avaliação do desempenho e efetividade da Estratégia Saúde da Família e da incorporação das ações de alimentação e nutrição". Trata-se de um estudo de corte transversal de abordagem quantitativa com coleta de dados primários obtidos no período de junho a dezembro de 2010, no município de Campina Grande (PB).

A população de estudo foi composta por enfermeiros da ESF e profissionais de saúde dos NASF da cidade de Campina Grande (PB). Considerou-se a inclusão de $30 \%$ dos enfermeiros $(n=20)$, selecionados aletoriamente, e de todos os profissionais dos NASF $(\mathrm{n}=75)$. Excluíram-se profissionais dos NASF com vínculo laboral inferior aos seis meses $(n=3)$, ponderando-se a questão da estabilidade, aqueles que estavam de férias ou de licença médica/ maternidade $(n=11)$ e os que não possuíam contato telefônico disponível ( $n=7)$. Esses mesmos critérios de exclusão foram considerados para o caso dos enfermeiros, entretanto nenhum deles foi enquadrado nestes. Não aceitaram participar da pesquisa 22 dos profissionais dos NASF. Assim, foram entrevistados nove fisioterapeutas, um médico, cinco assistentes sociais, cinco farmacêuticos, quatro educadores físicos, quatro psicólogos, dois nutricionistas e dois fonoaudiólogos. Dessa forma, a amostra total foi constituída por 52 profissionais.

Para a coleta de dados, utilizou-se um questionário estruturado com informações sobre formação profissional, características do trabalho e satisfação profissional. As variáveis de estudo foram escolhidas considerando a literatura revisada. $\mathrm{Na}$ formação profissional foi abordado o tipo de graduação, a realização de curso de pós-graduação e os cursos de capacitação frequentados. Nas características do trabalho consideraram-se informações sobre a forma de ingresso, o regime de trabalho, a satisfação com o tipo de vínculo empregatício, a carga horária, o recebimento de salário com atraso, a existência de incentivo salarial e possuir ou não outro vínculo empregatício. Ainda, os profissionais foram questionados sobre as principais atividades desenvolvidas na rotina de trabalho, em relação às relações humanas e a respeito do desempenho.

A análise da satisfação profissional compreendeu os seguintes aspectos: estrutura física, atendimento individual à demanda na unidade e no domicílio, trabalho em equipe, trabalho burocrático e participação em reuniões. Os profissionais pontuaram esses critérios de zero (pouco satisfeito) a 10 (muito satisfeito).

As variáveis relacionadas à formação profissional e características do trabalho foram apresentadas em tabelas de frequências absolutas e relativas. Para a satisfação profissional utilizaram-se escores médios e respectivos desvio padrão (DP).

Os dados coletados foram organizados em planilhas eletrônicas e digitados em dupla entrada no Excel. O aplicativo validate do software Epi Info versão 3.3.2 foi usado para analisar a consistência dos dados. As análises dos dados foram realizadas com o software Stata versão 12.0. 
O projeto foi avaliado e aprovado pelo Comitê de Ética em Pesquisa da Universidade Estadual da Paraíba (parecer $n^{\circ}$ 0355013300009), respeitando as diretrizes da Resolução 196/96 do Conselho Nacional de Saúde. Após os esclarecimentos, os sujeitos assinaram um termo de Consentimento Livre e Esclarecido, sendo garantido o sigilo das informações.

\section{RESULTADOS}

Entre os enfermeiros, 19 (95\%) eram do sexo feminino com idade média de 33 anos ( $\mathrm{DP}=8,7$ anos). Entre os profissionais dos NASF, 27 (84,4\%) eram do sexo feminino com idade média de 34,9 anos ( $\mathrm{DP}=8,4$ anos).

As características da formação dos enfermeiros e profissionais dos NASF estão descritas na Tabela 1. Observa-se que 16 enfermeiros $(80 \%)$ e 25 profissionais dos NASF $(75,1 \%)$ tinham especialização. Entretanto, apenas quatro enfermeiros (20\%) tinham mestrado. Em um contexto geral, a frequência a cursos foi maior entre enfermeiros do que nos profissionais dos NASF. Os cursos de tuberculose foram os mais frequentados pelos enfermeiros (85\%), enquanto o curso introdutório foi o mais frequentado pelos profissionais que atuavam nos NASF $(81,2 \%)$. Entre os enfermeiros, apenas oito (40\%) frequentaram cursos sobre saúde do adulto e saúde da criança e do adolescente, sendo esses os cursos menos frequentados. Nenhum dos profissionais dos NASF frequentou os cursos de hanseníase e de tuberculose.

No que concerne às características gerais do trabalho (Tabela 2), nota-se que todos os enfermeiros eram concursados com regime de trabalho estatutário e carga horária semanal de

Tabela 1: Características da formação de enfermeiros da Estratégia de Saúde da Família (ESF) e profissionais de saúde de Núcleos de Apoio à Saúde da Família (NASF) de Campina Grande, Paraíba, 2010

\begin{tabular}{|c|c|c|c|c|}
\hline \multirow[t]{2}{*}{ Características da formação } & \multicolumn{2}{|c|}{$\begin{array}{l}\text { Enfermeiros } \\
\text { da ESF }\end{array}$} & \multicolumn{2}{|c|}{$\begin{array}{l}\text { Profissionais } \\
\text { de NASF }\end{array}$} \\
\hline & $\mathbf{n}$ & $\%$ & $\mathbf{n}$ & $\%$ \\
\hline \multicolumn{5}{|l|}{ Pós-graduação } \\
\hline $\begin{array}{l}\text { Residência médica ou de } \\
\text { enfermagem }\end{array}$ & 1 & 5,0 & 1 & 3,1 \\
\hline Especialização & 16 & 80,0 & 25 & 78,1 \\
\hline Mestrado & 4 & 20,0 & - & - \\
\hline Doutorado & - & - & - & - \\
\hline \multicolumn{5}{|l|}{ Cursos de capacitação } \\
\hline Introdutório & 14 & 70,0 & 26 & 81,2 \\
\hline $\begin{array}{l}\text { Preenchimento do Sistema } \\
\text { de Informação da Atenção } \\
\text { Básica }\end{array}$ & 10 & 50,0 & 12 & 37,5 \\
\hline $\begin{array}{l}\text { Saúde da criança e do } \\
\text { adolescente }\end{array}$ & 8 & 40,0 & 17 & 53,1 \\
\hline Saúde da mulher & 16 & 80,0 & 16 & 50,0 \\
\hline Saúde do adulto & 8 & 40,0 & 4 & 12,5 \\
\hline Diabetes & 10 & 50,0 & 5 & 15,6 \\
\hline Hipertensão & 11 & 55,0 & 4 & 12,5 \\
\hline IST/AIDS & 14 & 70,0 & 12 & 37,5 \\
\hline Hanseníase & 13 & 65,0 & - & - \\
\hline Tuberculose & 17 & 85,0 & - & - \\
\hline
\end{tabular}

IST: Infecções Sexualmente Transmissíveis.
40 horas. A maioria deles (95\%) manifestou estar satisfeito com o tipo de vínculo empregatício. Entre os profissionais dos NASF, observaram-se como características predominantes a indicação $(59,4 \%)$ e a seleção interna da instituição $(31,3 \%)$ como formas de ingresso, o contrato temporário $(71,9 \%)$ e a carga horária de 40 horas semanais $(68,8 \%)$. Manifestaram insatisfação com a forma de vínculo empregatício 17 profissionais dos NASF $(53,1 \%)$. $\mathrm{O}$ incentivo salarial e o atraso no salário foram práticas pouco relatadas. A realização de outras atividades laborais foi apontada por nove enfermeiros (45\%) e 11 profissionais dos NASF (34,4\%).

Durante uma semana típica de trabalho, as principais atividades, por ordem, que os enfermeiros relataram realizar foram: atendimento individual, visita domiciliar e tarefas burocráticas. Os profissionais dos NASF destacaram as reuniões em equipe, a visita domiciliar e o atendimento individual. As reuniões com o nível central da Secretaria de Saúde e as atividades de capacitação

Tabela 2: Características do trabalho de enfermeiros da Estratégia de Saúde da Família (ESF) e profissionais de saúde de Núcleos de Apoio à Saúde da Família (NASF) de Campina Grande, Paraíba, 2010

\begin{tabular}{|c|c|c|c|c|}
\hline \multirow[t]{2}{*}{ Características do trabalho } & \multicolumn{2}{|c|}{$\begin{array}{l}\text { Enfermeiros } \\
\text { da ESF }\end{array}$} & \multicolumn{2}{|c|}{$\begin{array}{l}\text { Profissionais } \\
\text { de NASF }\end{array}$} \\
\hline & $\mathbf{n}$ & $\%$ & $\mathbf{n}$ & $\%$ \\
\hline \multicolumn{5}{|l|}{ Características gerais } \\
\hline \multicolumn{5}{|l|}{ Forma de ingresso } \\
\hline Concurso público & 20 & 100,0 & 1 & 3,1 \\
\hline $\begin{array}{l}\text { Seleção interna na } \\
\text { instituição }\end{array}$ & - & - & 10 & 31,3 \\
\hline Seleção externa & - & - & 2 & 6,3 \\
\hline Indicação & - & - & 19 & 59,4 \\
\hline \multicolumn{5}{|l|}{ Regime de trabalho } \\
\hline Estatutário & 20 & 100,0 & 1 & 3,1 \\
\hline CLT & - & - & 7 & 21,9 \\
\hline Contrato temporário & - & - & 23 & 71,9 \\
\hline Contrato informal & - & - & 1 & 3,1 \\
\hline Cargo comissionado & - & - & - & - \\
\hline $\begin{array}{l}\text { Satisfação com a forma de } \\
\text { vínculo }\end{array}$ & 19 & 95,0 & 15 & 46,9 \\
\hline \multicolumn{5}{|l|}{ Carga horária } \\
\hline 20 horas semanais & - & - & 10 & 31,3 \\
\hline 40 horas semanais & 20 & 100,0 & 22 & 68,8 \\
\hline $\begin{array}{l}\text { Pontualidade no recebimento } \\
\text { do salário }\end{array}$ & 18 & 90,0 & 32 & 100,0 \\
\hline Incentivo salarial do município & 3 & 15,0 & 1 & 3,1 \\
\hline Vínculo empregatício único & 11 & 55,0 & 21 & 65,6 \\
\hline \multicolumn{5}{|c|}{ Principais atividades em uma semana típica de trabalho } \\
\hline Visita domiciliar & 19 & 95,0 & 27 & 84,4 \\
\hline Atendimento individual & 20 & 100,0 & 27 & 84,4 \\
\hline $\begin{array}{l}\text { Coordenação/participação } \\
\text { em grupo terapêutico }\end{array}$ & 9 & 45,0 & 23 & 71,9 \\
\hline Reuniões em equipe & 12 & 60,0 & 30 & 93,7 \\
\hline $\begin{array}{l}\text { Reuniões com a } \\
\text { comunidade }\end{array}$ & 5 & 25,0 & 20 & 62,5 \\
\hline $\begin{array}{l}\text { Reuniões com o nível } \\
\text { central da Secretaria de } \\
\text { Saúde }\end{array}$ & 3 & 15,0 & 9 & 28,1 \\
\hline $\begin{array}{l}\text { Tarefas burocráticas } \\
\text { (relatórios, pedidos) }\end{array}$ & 18 & 90,0 & 17 & 53,13 \\
\hline $\begin{array}{l}\text { Participação em atividades } \\
\text { de capacitação }\end{array}$ & 3 & 15,0 & 10 & 31,25 \\
\hline
\end{tabular}


foram constatadas como práticas incomuns por ambos os grupos de profissionais. Adicionalmente, poucos enfermeiros citaram realizar reuniões com a comunidade (Tabela 2).

A remuneração média foi de $\mathrm{R} \$ 2362,21$ e $\mathrm{R} \$ 1276,6$ para enfermeiros e profissionais dos NASF, respectivamente. Para os enfermeiros, o período de trabalho junto às suas equipes foi de 14,7 meses ( $\mathrm{DP}=3,3$ anos); e para os profissionais dos NASF, 15,1 meses ( $\mathrm{DP}=3,8$ meses). Mudanças nas equipes de trabalho no último ano foram indicadas por oito enfermeiros (40\%) e $30(93,8 \%)$ dos profissionais dos NASF (dados não inclusos em tabela).

A opinião dos enfermeiros e dos profissionais dos NASF em relação ao trabalho encontra-se mostrada na Tabela 3. Os aspectos destacados como deficitários por ambos os grupos foram: falta de recursos, falta de capacitação e diversidade de tarefas. Comparativamente, os enfermeiros advertiram limitações para uma maior quantidade de itens do que os profissionais dos NASF, com destaque para a não concordância com o andamento do trabalho (60\%) e o excesso de atividades burocráticas (90\%).

Tabela 3: Opinião em relação ao trabalho de enfermeiros da Estratégia Saúde da Família (ESF) e profissionais de saúde de Núcleos de Apoio à Saúde da Família (NASF) de Campina Grande, Paraíba, 2010

\begin{tabular}{|c|c|c|c|c|}
\hline \multirow{3}{*}{ Aspectos avaliados } & \multicolumn{4}{|c|}{ Respostas positivas } \\
\hline & \multicolumn{2}{|c|}{$\begin{array}{c}\text { Enfermeiros da } \\
\text { ESF }\end{array}$} & \multicolumn{2}{|c|}{$\begin{array}{l}\text { Profissionais } \\
\text { de NASF }\end{array}$} \\
\hline & $\mathbf{n}$ & $\%$ & $\mathbf{n}$ & $\%$ \\
\hline $\begin{array}{l}\text { Muita concorrência } \\
\text { entre colegas }\end{array}$ & 1 & 5,0 & 1 & 3,1 \\
\hline $\begin{array}{l}\text { Falta de solidariedade } \\
\text { entre colegas }\end{array}$ & 11 & 55,0 & 7 & 21,9 \\
\hline $\begin{array}{l}\text { Relacionamento tenso } \\
\text { com os colegas }\end{array}$ & 2 & 10,0 & 1 & 3,1 \\
\hline Descontente com os colegas & 6 & 30,0 & 1 & 3,1 \\
\hline $\begin{array}{l}\text { Facilidade de dialogar } \\
\text { com chefias }\end{array}$ & 17 & 85,0 & 29 & 90,6 \\
\hline $\begin{array}{l}\text { Relacionamento tenso } \\
\text { com chefias }\end{array}$ & 2 & 10,0 & 2 & 6,2 \\
\hline $\begin{array}{l}\text { Recursos carentes } \\
\text { para o trabalho }\end{array}$ & 20 & 100,0 & 29 & 90,6 \\
\hline $\begin{array}{l}\text { Falta de capacitação } \\
\text { para as tarefas }\end{array}$ & 17 & 85,0 & 23 & 71,9 \\
\hline Reconhecimento pelo trabalho & 9 & 45,0 & 23 & 71,9 \\
\hline $\begin{array}{l}\text { Concordância com o } \\
\text { andamento do trabalho }\end{array}$ & 8 & 40,0 & 20 & 62,5 \\
\hline Compromisso com o trabalho & 20 & 100,0 & 32 & 100,0 \\
\hline Ser útil no trabalho & 19 & 95,0 & 32 & 100,0 \\
\hline Orgulho pelo trabalho & 19 & 95,0 & 32 & 100,0 \\
\hline Exigência excessiva & 11 & 55,0 & 2 & 6,2 \\
\hline Muita responsabilidade & 16 & 80,0 & 13 & 40,6 \\
\hline $\begin{array}{l}\text { Excesso de trabalho } \\
\text { burocrático }\end{array}$ & 18 & 90,0 & 5 & 15,6 \\
\hline Diversidade de tarefas & 20 & 100,0 & 27 & 84,4 \\
\hline Insegurança pela instabilidade & 0 & 0,0 & 20 & 62,5 \\
\hline Medo de ficar sem trabalho & 1 & 5,0 & 17 & 53,1 \\
\hline $\begin{array}{l}\text { Liberdade para } \\
\text { sugerir melhorias }\end{array}$ & 19 & 95,0 & 31 & 96,9 \\
\hline $\begin{array}{l}\text { Possibilidade de aplicar } \\
\text { os conhecimentos }\end{array}$ & 20 & 100,0 & 29 & 90,6 \\
\hline Possibilidade de progredir & 11 & 55,0 & 28 & 87,5 \\
\hline
\end{tabular}

A Tabela 4 apresenta os escores médios de satisfação no trabalho. Tanto enfermeiros quanto profissionais dos NASF indicaram menor satisfação para estrutura física $(4,8 \pm 2,0$ e 4,3 $\pm 2,0)$, atendimento individual à demanda na unidade de saúde $(5,9 \pm 1,6$ e $6,0 \pm 2,0)$ e reuniões com a comunidade $(6,0 \pm 2,5$ e $6,5 \pm 2,3)$. Reuniões com a coordenação local representou o aspecto de maior satisfação tanto para enfermeiros $(6,8 \pm 1,3)$ quanto para profissionais dos NASF $(7,3 \pm 1,6)$, seguido pelo trabalho em equipe $(7,2 \pm 2,2)$ e reuniões de equipe $(7,1 \pm 2,5)$, no caso dos profissionais dos NASF; e pelo atendimento individual à demanda $(6,4 \pm 1,3)$ e preenchimento de formulários e relatórios no domicílio $(6,4 \pm 2,2)$, no caso dos enfermeiros.

\section{DISCUSSÃO}

Neste estudo, a caracterização do trabalho de 52 profissionais da APS permite sugerir a estabilidade profissional como condição relacionada à satisfação empregatícia.

Visita domiciliar e atendimento individual são as atividades mais rotineiras desses profissionais, os quais destacam a falta de recursos, a falta de capacitação e a diversidade de tarefas como principais aspectos negativos. A maioria dos enfermeiros indicou falta de solidariedade, não reconhecimento pelo trabalho, não concordância com o andamento do trabalho, exigência excessiva, muita responsabilidade e excesso de trabalho burocrático. Insegurança pela instabilidade e medo de ficar sem trabalho também apresentaram altas frequências entre os profissionais dos NASF. Do ponto de vista de satisfação, destacou-se o descontentamento com a estrutura física, o atendimento individual à demanda na unidade de saúde e as reuniões com a comunidade.

Percebe-se que a maioria dos enfermeiros da ESF de Campina Grande (PB) tem idade próxima dos 30 anos, resultado semelhante ao encontrado em outros estudos ${ }^{9,10}$. A predominância do profissional de enfermagem de sexo feminino, similar ao

Tabela 4: Escores médios de satisfação com o trabalho de enfermeiros da Estratégia Saúde da Família (ESF) e profissionais de saúde de Núcleos de Apoio à Saúde da Família (NASF) de Campina Grande, Paraíba, 2010

\begin{tabular}{|c|c|c|}
\hline \multirow[t]{2}{*}{ Variáveis de satisfação } & $\begin{array}{l}\text { Enfermeiros } \\
\text { da ESF }\end{array}$ & $\begin{array}{l}\text { Profissionais } \\
\text { de NASF }\end{array}$ \\
\hline & Média $( \pm \mathrm{DP} *)$ & Média ( $\left( \pm \mathrm{DP}^{\star}\right)$ \\
\hline $\begin{array}{l}\text { Estrutura física da } \\
\text { unidade de saúde }\end{array}$ & $4,8( \pm 2,0)$ & $4,3( \pm 2,0)$ \\
\hline $\begin{array}{l}\text { Atendimento individual à } \\
\text { demanda na unidade de saúde }\end{array}$ & $5,9( \pm 1,6)$ & $6,0( \pm 2,0)$ \\
\hline $\begin{array}{l}\text { Atendimento individual à } \\
\text { demanda no domicílio }\end{array}$ & $6,4( \pm 1,3)$ & $6,6( \pm 1,8)$ \\
\hline Trabalho em equipe & $6,3( \pm 2,2)$ & $7,2( \pm 2,2)$ \\
\hline $\begin{array}{l}\text { Preenchimento de } \\
\text { formulários e relatórios }\end{array}$ & $6,4( \pm 2,2)$ & $6,6( \pm 2,1)$ \\
\hline Reuniões de equipe & $6,1( \pm 2,5)$ & $7,1( \pm 2,5)$ \\
\hline Reuniões com a comunidade & $6,0( \pm 2,5)$ & $6,5( \pm 2,3)$ \\
\hline $\begin{array}{l}\text { Reuniões com a } \\
\text { coordenação local }\end{array}$ & $6,8( \pm 1,3)$ & $7,3( \pm 1,6)$ \\
\hline
\end{tabular}

${ }^{*} \mathrm{DP}=$ desvio padrão. 
presente estudo, foi anteriormente visto para a região Nordeste do país ${ }^{9}$ e no município de Cascavel $(\mathrm{PR})^{11}$. Esses resultados não fogem das características da força de trabalho integrada aos NASF em Campina Grande, composta, em sua expressiva maioria, por mulheres.

Neste trabalho, a frequência de profissionais que tinham curso de especialização foi maior do que o resultado encontrado no âmbito nacional e na região Nordeste do Brasil'. Resultado positivo também foi encontrado em um estudo realizado com enfermeiros das equipes de Saúde da Família de Cuiabá (MT), no qual se verificou que todos os sujeitos cursaram ou estavam cursando pós-graduação lato sensu, em sua maioria voltada à Saúde Pública ${ }^{12}$. Esses achados são relevantes, pois cursar pós-graduação na área da saúde pode influenciar positivamente as práticas profissionais ${ }^{13}$.

O trabalho na ESF, além do conhecimento técnico específico, requer dos profissionais, entre outros, conhecimentos sobre as políticas públicas de saúde e do perfil epidemiológico da população ${ }^{7}$. Em contraponto, estudo realizado na região Nordeste relatou ser reduzido o número de profissionais de saúde da ESF com formação na área de Saúde Pública ${ }^{9}$. Na América Latina situação similar foi encontrada ao destacar a carência de enfermeiros com formação específica para atuar no cuidado na APS e as dificuldades no monitoramento dos cursos de pós-graduação no que se refere à formação assistencial e acadêmica desses profissionais ${ }^{14}$.

Estudo desenvolvido em Vitória (ES) ${ }^{3}$ abordando a frequência em cursos de capacitação por enfermeiros da ESF encontrou os melhores resultados para hanseníase, IST/AIDS (Infecções Sexualmente Transmissíveis/AIDS), tuberculose, dengue e imunização. Os autores destacaram a participação de enfermeiros em cursos de capacitação como forma de melhorar as ações da ESF. É necessário que a educação permanente em saúde ganhe espaço como política pública flexível e não fragmentada, considerada com a devida importância pelos profissionais para a transformação das práticas de saúde ${ }^{15}$. No presente estudo, os profissionais também relataram ter frequentado cursos sobre tuberculose, imunização e IST/AIDS, bem como destacaram a influência positiva das capacitações para o desenvolvimento de suas atividades, conforme relatado por 19 (95\%) dos enfermeiros da ESF e 25 $(78,1 \%)$ dos profissionais dos NASF. Os enfermeiros contextualizaram benefícios relacionados ao aperfeiçoamento, atualização do conhecimento, melhora do atendimento e qualidade da prática assistencial. Para os profissionais dos NASF, as capacitações influenciaram positivamente o trabalho devido às possibilidades de atualizar o conhecimento, melhorar a qualidade do serviço prestado e aperfeiçoar os procedimentos em equipe.

Neste estudo, a percentagem de enfermeiros $(55,0 \%)$ e profissionais dos NASF $(65,6 \%)$ que relataram ter outro vínculo empregatício não foi observada em resultados anteriores ${ }^{11,16}$. Em municípios com mais de 100 mil habitantes da região Nordeste, a maioria dos profissionais da ESF trabalhavam em apenas uma instituição ${ }^{16}$, mas neste estudo foram considerados trabalhadores de todas as profissões o que possivelmente pode explicar essa diferença. Entretanto, resultado semelhante aos constatados em Campina Grande foram descritos também no município de Floriano (PI), no qual a maioria dos enfermeiros da ESF apresentava outro vínculo, ressaltando-se a necessidade de dois ou mais trabalhos em virtude da ausência de um salário compatível com a formação e competência profissional ${ }^{10}$. Baixa remuneração, falta de motivação dos profissionais, precariedade dos vínculos empregatícios e dificuldades na implementação de estratégias para a redução da rotatividade dos trabalhadores são pontos críticos que vêm sendo debatidos no contexto do sistema de saúde brasileiro que precisam de soluções urgentes ${ }^{17}$.

Estudo que abordou a situação salarial dos enfermeiros das regiões Nordeste e Sul apontou ausência de diferença, sendo os rendimentos médios destes profissionais de R $\$ 2399$ e R \$ 2211, respectivamente ${ }^{16}$. Esses resultados são similares aos encontrados no atual estudo e revertem-se de especial importância ao considerar que a estabilidade profissional pode repercutir positivamente nas práticas e gerar autonomia para realizar mudanças na gestão do serviço, na rotina da unidade e na assistência prestada ${ }^{18}$.

Em relação às atividades desenvolvidas em uma semana típica de trabalho, a menor frequência de atividades coletivas entre os enfermeiros do que nos profissionais dos NASF pode ser uma dificuldade relacionada com a reorganização da assistência centrada na prevenção de agravos e na promoção da saúde coletivo-individual. As ações coletivas predispõem um trabalho multiprofissional, tendo a possibilidade de quebrar a divisão de trabalho em saúde segundo recortes verticais e centradas no médico². No NASF, as ações devem considerar o trabalho em conjunto com as equipes da ESF, com vista a qualificar e complementar as ações de forma compartilhada para superar a lógica fragmentada ainda hegemônica no cuidado à saúde ${ }^{19}$.

A carga de trabalhos burocráticos como problemática também ressaltada entre os enfermeiros deste estudo pode ocasionar dificuldades no cumprimento e reflexão sobre as ações ${ }^{3}$. Pesquisas anteriores apontaram similarmente excesso de atividades administrativas, burocráticas e gerenciais vinculadas às ações de enfermagem ${ }^{5,20,21}$.

Nos territórios da ESF, as equipes convivem com dezenas de formulários que, em geral, estão sob a responsabilidade dos enfermeiros, fazendo com que estes profissionais fiquem sobrecarregados pelo excesso de burocracia. Este acúmulo de atividades gera um ambiente de trabalho precário e de práticas profissionais irregulares. Por consequência, há prejuízos sobre os direitos e as garantias dos trabalhadores, contribuindo com a falta de estímulo para a execução das atividades e sofrimento do profissional em seu ambiente de trabalho ${ }^{20-22}$.

Destaca-se, ainda, a sobrecarga de atividades dos enfermeiros como fator prejudicial à produção de autonomia e consequente reconhecimento profissional ${ }^{23}$. Associado ao excesso de burocracia, a carga de trabalho excessiva, a insegurança profissional, e a necessidade de qualificação e de realização própria, foram aspectos destacados por enfermeiros do Ceará ao avaliar a percepção 
acerca de sua prática na ESF, tornando-se evidente a necessidade da criação de uma política trabalhista para ESF associada à valorização dos trabalhadores ${ }^{20}$.

Coerentemente com o vínculo empregatício, muitos profissionais dos NASF indicaram, diferente dos enfermeiros, insegurança pela instabilidade e medo de perder o trabalho. Nesse sentido, salienta-se a importância da fixação dos profissionais nos serviços como forma de melhores garantias trabalhistas e menor precarização do trabalho ${ }^{24}$. Diante dos resultados considera-se a necessidade de vínculos trabalhistas mais estáveis para os profissionais dos NASF. Ressalta-se a importância de concursos públicos e salários que estimulem a qualificação desses profissionais. Por serem profissionais com carga horária inferior a 40 horas semanais, deduz-se que os trabalhadores dos NASF têm maior facilidade a agregarem outros vínculos empregatícios a sua jornada de trabalho.

A sobrecarga de trabalho e as condições laborais inadequadas são possíveis fatores geradores de estresse emocional e físico, que podem prejudicar a saúde e o desempenho profissional com consequências no cuidado ${ }^{25}$. Esses fatores tiveram alta proporção de avaliação negativa entre os enfermeiros estudados, o que sugere a necessidade de melhorias nas condições de trabalho. A implementação de Planos de Cargos e Carreiras e Remuneração para todos os profissionais da saúde pode ser um mecanismo viável que ajude no enfrentamento desses desafios, contribuindo de maneira positiva nas disparidades regionais e na valorização do desempenho.

A baixa satisfação dos profissionais de saúde deste estudo com a estrutura física das unidades de saúde coincide com resultados divulgados anteriormente ${ }^{26}$. Trabalhar em condições desumanas, associadas à falta de recursos, sobrecarga de trabalho e outros fatores podem colocar os profissionais de saúde em situações de risco, vulnerabilidade e incapacidade ${ }^{27}$. A satisfação no trabalho influencia o comportamento do trabalhador e a qualidade de vida do indivíduo ${ }^{26}$.

As limitações do atual estudo incluem sua ênfase descritiva relacionada a um único município e amostra por conveniência que restringe seu poder de generalização. Deve ser destacado também que se baseou exclusivamente nas respostas dos profissionais com possibilidade de viés de informação. Ainda, há que mencionar que foram observados apenas enfermeiros e profissionais de saúde de NASF, não representando os resultados a conjuntura de profissionais da APS de modo geral. Outra limitação relaciona-se ao tempo de coleta dos dados, desfasados em sete anos. Entretanto, a comparação dos resultados com estudos atuais possibilitou verificar que não houve mudanças nas características do trabalho, com concepção de mérito. Apesar dessas limitações os achados apresentam contribuição importante em dois âmbitos: para as políticas e formação de recursos humanos de enfermagem, uma das seis categorias principais de prioridades de pesquisa em enfermagem na América Latina ${ }^{28}$; e para a gestão do trabalho e educação em saúde, parte da agenda de prioridades de pesquisa em saúde no Brasil ${ }^{29}$.

O presente estudo possibilitou conhecer características do trabalho de enfermeiros da ESF e profissionais de saúde de NASF que são relevantes para subsidiar o planejamento dos serviços de saúde da APS no município estudado. Os resultados refletem dificuldades e insatisfação com as condições de trabalho que inclui um cenário estrutural das unidades de saúde pouco favorável e vínculos trabalhistas marcados por insegurança, falta de solidariedade e baixo reconhecimento. Esses achados podem auxiliar a organização do trabalho nas equipes de saúde da ESF e dos NASF dos quais esses profissionais fazem parte ao apontar fragilidades que precisam ser superadas.

\section{REFERÊNCIAS}

1. Brasil. Ministério da Saúde. Portaria $n^{\circ} 2.436$, de 21 de setembro de 2017. Aprova a Política Nacional de Atenção Básica, estabelecendo a revisão de diretrizes para a organização da Atenção Básica, no âmbito do Sistema Único de Saúde (SUS). Diário Oficial República Federativa Brasil. 2017.

2. Brasil. Ministério da Saúde. Secretaria de Atenção à Saúde. Departamento de Atenção Básica Diretrizes do NASF: Núcleo de Apoio a Saúde da Família. Brasília: Ministério da Saúde; 2009.

3. Silva VG, Motta MCS, Zeitoune RCG. A prática do enfermeiro na Estratégia Saúde da Família: o caso do município de Vitória/ES. Rev Eletr Enf. 2010;12(3):441-8. http://dx.doi.org/10.5216/ree.v12i3.5278

4. Barbosa RC, Lorenzini E, Bazzo K, Silva EF. Liderança do enfermeiro na estratégia saúde da família: revisão integrativa. Rev Eletr Gestão Saúde. 2013;4(2):428-38. http://dx.doi.org/10.18673/gs.v4i2.22955
5. Galavote HS, Zandonade E, Garcia ACP, Freitas PSS, Seidl H, Contarato PC, et al. O trabalho do enfermeiro na atenção primária à saúde. Esc Anna Nery 2016;20(1):90-8. http://dx.doi.org/10.5935/1414-8145.20160013

6. Motta LCS, Siqueira-Batista R. Estratégia Saúde da Família: Clínica e Crítica. Rev Bras Educ Med. 2015;39(2):196-207. http://dx.doi.org/10.1590/1981-52712015v39n2e00912014

7. Santos MC, Boechat Frauches M, Rodrigues SM, Toledo FE. Processo de Trabalho do Núcleo de Apoio à Saúde da Família (NASF): Importância da Qualificação Profissional. Sau \& Transf Soc 2017;8(2):60-9.

8. Tambasco LP, Silva HS, Pinheiro KMK, Gutierrez BAO. A satisfação no trabalho da equipe multiprofissional que atua na Atenção Primária à Saúde. Saúde Debate 2017;41(N Especial):140-151. http://dx.doi.org/10.1590/0103-11042017s212 
9. Machado MH, Stiebler AL, Oliveira ES, Lampert J, D'Aguiar J, Silva $L$, et al. Perfil dos médicos e enfermeiros do Programa Saúde da Família no Brasil: relatório final. Brasília: Ministério da Saúde; 2000.

10. Rocha MCP, Martino MMF, Grassi-Kassisse DM, Souza AL. Estresse em enfermeiros: o uso do cortisol salivar no dia de trabalho e de folga. Rev Esc Enferm USP. 2013;47(5):1194-01. http://dx.doi.org/10.1590/S0080-623420130000500025

11. Roecker S, Nunes EFPA, Marcon SS. O trabalho educativo do enfermeiro na estratégia saúde da família. Texto Contexto Enferm. 2013;22(1):157-65 http://dx.doi.org/10.1590/S0104-07072013000100019

12. Duarte SJH, Ferreira SF, Santos NC. Desafios de enfermeiros da Estratégia Saúde da Família na implantação do Programa Saúde do Adolescente. Rev Eletr Enf. 2013; 15(2):479-86. http://dx.doi.org/10.5216/ree.v15i2.18179

13. Costa CMM, Chagas HMA, Matsukura TS, Vieira GI, Marqueze EC, López CG, et al. Contribuições da pós-graduação na área da saúde para a formação profissional: relato de experiência. Saúde Soc. 2014;23(4):1471-81. http://dx.doi.org/10.1590/S0104-12902014000400028

14. Ceitlin J. La medicina familiar en América Latina. Presentación. Aten Primaria. 2006;38(9):511-4. https://dx.doi.org/10.1157/13095056

15. Barth PO, Aires M, Santos JLG, Ramos FRS. Educação permanente em saúde: concepções e práticas de enfermeiros de unidades básicas de saúde. Rev Eletr Enf. 2014;16(3):604-11. http://dx.doi.org/10.5216/ree.v16i3.22020

16. Tomasi E, Facchini LA, Piccini RX, Thumé E, Silveira DS, Siqueira FV, et al. Perfil sócio demográfico e epidemiológico dos trabalhadores da atenção básica à saúde nas regiões Sul e Nordeste do Brasil. Cad Saúde Pública. 2008;24(1):193-201. http://dx.doi.org/10.1590/S0102-311X2008001300023

17. Vieira SP, Pierantoni SR, Magnago C, França T, Miranda RG. Planos de carreira, cargos e salários no âmbito do Sistema Único de Saúde: além dos limites e testando possibilidades. Saúde Debate. 2017;41(112):110-21.

http://dx.doi.org/10.1590/0103-1104201711209

18. Raimundi DM, Ferreira FF, Lima FCA, Siqueira VCA. Análise de uma Clínica da Família, visão dos enfermeiros do serviço. Rev Esc Enferm USP. 2016;50(n.spe):130-8.

http://dx.doi.org/10.1590/S0080-623420160000300019
19. Albuquerque IMAN, Araujo Neto JD, Lira GV, Ribeiro MA, Santos FD, Vasconcelos MIO. Contribuições do Núcleo de Apoio à Saúde da Família quanto ao princípio da integralidade. Investigação Qualit Saúde. 2016;2:1482-91.

20. Ximenes Neto FRG, Ponte MAC, Amaral MIV, Rocha J, Cunha ICKO. Percepção dos enfermeiros sobre a prática profissional na estratégia saúde da família. Disponível em: http://www.index-f. com/lascasas/documentos/lc0468.php. Aceso em: 07 jul. 2017.

21. Souza MG, Mandu ENT, Elias AN. Percepções de enfermeiros sobre seu trabalho na estratégia saúde da família. Texto Contexto Enferm. 2013;22(3):772-9. http://dx.doi.org/10.1590/S0104-07072013000300025

22. David HMSL, Mauro MYC, SilvaVG, Pinheiro MAS, SilvaFH. Organização do trabalho de enfermagem na Atenção Básica: uma questão para a saúde do trabalhador. Texto Contexto Enferm. 2009;18(2):206-14

23. Caçador BS, Lopes FN, Pacheco LC, Alves MS, Salimena AMO. O enfermeiro na estratégia de saúde da família: percepção dos usuários. HU Rev. 2012;37(3):331-8.

24. Santini SML, Nunes EFPA, Carvalho BG, Souza FEA. Dos 'recursos humanos' à gestão do trabalho: uma análise da literatura sobre o trabalho no SUS. Trabalho, Educação e Saúde. 2017;15(2):537-59. http://dx.doi.org/10.1590/1981-7746-sol00065

25. Silva DSD, Tavares NVS, Alexandre ARG, Freitas DA, Brêda MZ, Albuquerque MCS, et al. Depressão e risco de suicídio entre profissionais de Enfermagem: revisão integrativa. Rev Esc Enferm USP. 2015;49(6): 1027-36. http://dx.doi.org/10.1590/S0080-623420150000600020

26. Lima L, Pires DEP, Forte ECN, Medeiros F. Satisfação e insatisfação no trabalho de profissionais de saúde da atenção básica. Esc Anna Nery. 2014;18(1):17-24.

http://dx.doi.org/10.5935/1414-8145.20140003

27. Silva CCS, Rodrigues LMC, Silva VKBA, SilvaACO, Silva VLA, Martins MO. Percepção da enfermagem sobre condições de trabalho em unidades de saúde da família na Paraíba - Brasil. Rev Eletr Enf. 2013;15(1):205-14. http://dx.doi.org/10.5216/ree.v15i1.15074

28. Cassiani SHB, Bassalobre-Garcia A, Reveiz L. Acesso Universal à Saúde e Cobertura Universal de Saúde: identificação de prioridades de pesquisa em enfermagem na América Latina. Rev Latino-Am Enfermagem. 2015;23(6):1195-208. http://dx.doi.org/10.1590/0104-1169.1075.2667

29. Brasil. Ministério da Saúde. Agenda nacional de prioridades de pesquisa em saúde. 2 ed. 4 reimpr. Brasília: Ministério da Saúde; 2015. 